\title{
Challenging core cultural beliefs and maintaining the therapeutic alliance: A qualitative study
}

Word Count: 6622 words

Short Title: Challenging Core Cultural Beliefs

Key Words: culture, therapeutic alliance, family belief, qualitative research, black \& minority-ethnic client groups

Author details in separate document 


\title{
Challenging core cultural beliefs and maintaining the therapeutic alliance: A qualitative study
}

\author{
Challenging Core Cultural Beliefs
}

\begin{abstract}
(150 words)
Engaging black and minority-ethnic (BME) individuals in therapy and maintaining a positive therapeutic alliance can be a complex task, especially when challenging a family's core belief system. This study examines how therapists working within a specialist cultural service in London, UK were able to question a family's core cultural belief system whilst building and maintaining a strong therapeutic alliance. Video-assisted semi-structured interviews were carried out with two family members and their two therapists, and analysed using thematic analysis. The findings suggest that core beliefs can be effectively challenged within the context of a strong therapeutic alliance if topics are approached in a sensitive and respectful manner, by trusted therapists who have a degree of knowledge and awareness of their own cultural positioning, as well as an understanding of their client's culture. We propose that engaging with culture in this way can strengthen alliance and facilitate positive change.
\end{abstract}

\section{Practitioner Points (447 characters)}

- Core cultural belief systems can be effectively challenged by therapists within the context of a positive therapeutic alliance, if topics are approached sensitively with a degree of authenticity, curiosity and respect

- Respectfully challenging core beliefs whilst remaining aware of one's own cultural construction and positioning, as well as the client's, can strengthen therapeutic alliance and promote positive change 


\section{Introduction}

Providing effective mental health services to all members of a community can be challenging due to the diverse range of cultural differences and backgrounds. Therapists must work to provide clients with a valuable therapeutic experience that is mindful and aware of a client's cultural needs. Consideration of cultural factors is especially important when working with black and minority-ethnic (BME) client groups, as effective practice largely relies on the ability of the therapist to successfully engage a client in therapy by developing a strong therapeutic alliance (Asnaani \& Hofmann, 2012; Kirmayer, 2007). The processes by which family therapists develop positive therapeutic alliances with BME families in therapy is a topic of growing research interest, as the importance of considering a client's culture when delivering clinical services has become even greater within our increasingly diverse society. This has also, however, become more challenging within the contemporary social context where immigration has become a source of intense fear and BME communities, Muslims in particular, are being asked to integrate to British values (e.g. "Muslims must embrace British values", Telegraph, 2011). How then do we form and develop strong therapeutic alliances that facilitate integration and genuine change?

\section{Developing a positive alliance}

Therapeutic alliance can be defined as the quality and strength of the relationship between client and therapist, with an emphasis on collaboration and consensus (Horvath et al., 2011). Central to this process are the positive affective bonds that develop, including mutual trust, liking and respect (Horvath \& Bedi, 2002). Systematic reviews examining the key variables contributing to a positive therapeutic alliance agree that conveying empathy, warmth and genuineness towards a client are factors that largely influence the outcome of clinical practice, regardless of therapist orientation or the client's presenting problem (e.g. Elliott et al., 2011). The factors that foster a positive therapeutic alliance are important to consider in any clinical setting, but are especially significant when thinking about the engagement of BME clients in therapy. As a person's cultural values and belief system are central to the ways in which they conduct all aspects of their life, displaying empathy and an understanding of a family's culture during sessions is likely to impact on the effectiveness of therapy and strength of the therapeutic alliance (Asnaani \& Hofmann, 2012; Malik \& Mandin, 2012). Yet, this may be challenging for the therapist, especially when the client's beliefs or values are perceived as conflicting with our own. Finding a middle ground to make a genuinely collaborative relationship can be difficult. A recent study by Pandya and Herlihy (2009) looking at the therapeutic alliance as perceived by South Asian clients highlighted feelings of safety in therapy, working collaboratively with clients, and feeling cared for by the therapist as three key components to the development of a positive alliance with BME families. 


\section{Working with difference}

There is a general emphasis within existing literature on the need for therapists to align themselves with a client's viewpoint and accept their account of difficult race-related or culture-related events as 'the truth' in order to demonstrate full support for issues clients may have encountered, and to communicate empathy and encourage further discussion of sensitive topics (Vasquez, 2007). By remaining attuned to a client's personal situation, therapists are able to demonstrate a tolerance of all material that is brought to sessions, which can promote a feeling of respect and acceptance by providing clients with a safe and containing place to discuss all aspects of culture-specific material. Culture-specific material applies to the social context at the levels of individuals, families, communities and wider social systems (Kirmayer, 2012) in relation to a number of factors including ethnicity, race, religion, language, beliefs, social behaviour and customs. Factors such as the influence of cultural congruence on the client-therapist relationship (Tseng, 1999), the extent to which a therapist is knowledgeable about a client's cultural norms and culture-specific values such as family, respect and authority, and an understanding of a client's preferred way of relating interpersonally (e.g. hierarchical vs. collaborative, collectivist vs. individualistic) are all thought to impact the effectiveness of therapy and the development of a positive therapeutic alliance (Friedlander et al., 2006). A therapist's sensitivity towards a client's cultural circumstances (Lee, 2011) as well as a therapist's ability to be reflexive about their own cultural position (Krause, 2012) are also key to providing a safe and trusting space for a positive alliance to develop. It is therefore important for therapists to remain mindful of the specific values, prejudices and assumptions that both they and the family might hold and incorporate this knowledge into service delivery (Pandya \& Herlihy, 2009).

\section{Challenging core belief systems}

Consistent with the idea of remaining non-judgemental, the modus operandi seems to have been to avoid challenging the core cultural and religious beliefs that clients hold, in relation to their beliefs about themselves, others and the world, and how each interact. Research suggests that such challenges may be received as disrespectful or naïve (Asnaani \& Hofmann, 2012; Hays, 2009; Hofmann, 2006). Instead, it is recommended that the helpfulness or utility of a belief, rather than its validity, is questioned, to avoid the therapist making a determining statement regarding what they see as functional or rational in the client's culture (Beck, 2005). Recognising that ultimately it is the client, not the therapist, who is in the most knowledgeable position to decide whether a belief is helpful or unhelpful is also recommended (Wood \& Mallinckrodt, 1990).

The resistance to the challenging of core beliefs described within the literature could be linked to a fear of acting in a prejudiced or offensive manner, particularly at a crucial time where high sensitivity towards personal topics relating to culture, ethnicity or religion is recommended in order to develop an alliance. In some circumstances, challenging a client's belief system could also be considered ignorant, particularly if the therapist is from a different cultural background to the client. This research will explore the ways in which two 
therapists working within a specialist cultural service challenge the core cultural beliefs of a family undertaking therapy, and whether this can be done in a way that is compatible with creating and maintaining a positive therapeutic alliance.

\section{Methodology}

\section{The Setting}

The Marlborough Cultural Therapy Centre (MCTC) was a service specialising in the delivery of family therapy targeted towards black and minority-ethnic (BME) client groups. It was established in 1996 and disbanded in 2013 due to restructuring of National Health Service (NHS) Child and Adolescent Mental Health Services (CAMHS). The MCTC was based in Central London and was situated within The Marlborough Family Service ${ }^{1}$, part of a mainstream multi-disciplinary CAMHS. The MCTC consisted of a team of bilingual therapists, with spoken languages including Urdu, Bengali, Arabic, Mandarin and French. All therapists within the service were systemically trained, with specific training in developing culturally reflexive practice with children and families. The challenges involved in the development of such a culturally-focused service and its integration into mainstream CAMHS have been written about in more detail in Malik et al. (2009).

\section{The Family}

The case selected for this research involved a family undergoing therapy at the MCTC. The family, who had been undergoing therapy for around 18 months at the time that the research began, were purposively selected because they were a high risk case involving child protection issues and an alleged honour crime and fear of forced marriage, which related to challenges around gender and the struggle to negotiate different cultural contexts. The family felt that they had been subjected to racist assumptions and that whilst they acknowledged their son had behaved aggressively, they saw it as 'boys being boys' and 'things having got out of hand'. They were adamant there was no danger of them forcing their daughter into a marriage and they felt they were being treated harshly and made 'an example of'. The family were of Pakistani heritage and living in London. Both parents were in their mid-forties, and immigrated to the UK over 20 years ago with their eldest son to join relatives with the hope of improving their children's educational opportunities. They were from middle class families and suffered a significant loss in class status through the migration process as they had to settle for low-income employment and social housing. Their young adult sons (early twenties) and two teenage daughters who took part in family therapy sessions had been acutely aware of these differences. Work with this family therefore presented the therapists with the challenging task of helping the family find an area of shared understanding or middle ground, and provided an opportunity to explore how and whether it was possible to challenge core cultural beliefs both in the family and wider system in a sensitive manner. The

\footnotetext{
${ }^{1}$ Since this study was carried out, the Marlborough Family Service has merged with another CAMHS, and renamed Westminster CAMHS
} 
family, despite feeling subjected to racism, when met with empathy had engaged well with the service, and reported a strong sense of trust in the therapists as well as a good therapeutic alliance. The case therefore provided an opportunity to better understand how it might be possible to challenge core cultural beliefs in family therapy, whilst maintaining a strong sense of trust and alliance.

Having received ethical approval for the study from University College London (UCL) and the NHS Research Ethics Committee (REC), the family were approached by the therapists and given information about the study, and then invited to participate. Due to their availability at the time when interviews took place, the family members participating in the qualitative interviews conducted as part of this research were the father (Sajid) and youngest daughter (Farah, 15 yrs) who was born in the UK and spoke English and Urdu. Names and some details about the family have been changed for confidentiality purposes.

\section{The Therapists}

The therapists involved in this research, who are also co-authors in this paper, were the two professionals working jointly with the family within the MCTC. Therapist 1 describes herself as a Muslim British Pakistani woman, and has practised as a systemic family therapist for a number of years. She was born in Pakistan but moved to the UK at a young age, considering herself to be British yet very connected to her Pakistani roots. Therapist 2 describes himself as a white French Agnostic man who has lived in London longer than in France. He originally trained as a social worker, before moving into systemic family therapy. The therapists have been working together for over 10 years and specialise in working with clients of different cultural backgrounds. A culturally-engaged systemic approach to family therapy was adopted during the work with this family (see Malik \& Mandin, 2012 for a detailed description of the approach).

\section{The Researchers}

The first author of this paper, Researcher 1, who conducted this research as part of her MSc studies, describes herself as white British and has always lived in the UK. Her parents were both born in England; her father to Polish parents and her mother to a Spanish mother and British father. At the time of conducting this research she was working as an Assistant Psychologist at the MCTC as part of her MSc studies. The fourth author of this paper and supervisor of this research, Researcher 2, is a child psychotherapist and university lecturer. He describes himself as white British and has lived most of his life in the UK. His mother's family were Jewish immigrants from Eastern Europe and he himself is married to someone from East Asia, with a child who holds dual nationality and considers himself equally belonging to both cultures.

\section{Design}

Two semi-structured interviews were carried out by Researcher 1 in order to explore in-depth the factors that contributed towards building a positive therapeutic alliance with the family, in 
the context of challenging core cultural beliefs. The first interview involved the two family therapists, and the second the two family members. The questions asked related to the rationale behind the specific techniques adopted by the therapists, and the family's view of the therapists' approach.

The Interpersonal Process Recall (IPR) method was incorporated into the interviews. IPR is a qualitative interviewing technique that makes use of video-assisted recall to facilitate the identification of thoughts and feelings that occur during therapeutic interactions (Kagan, 1980). Researcher 1and Therapist 1 reviewed video-recordings of therapy sessions that had already been collected for clinical purposes, and identified two culturally-significant moments in therapy. These clips were then played to participants during both interviews in order to facilitate recall and encourage 'in-the-moment' reflections on specific moments in therapy.

\section{Data Analysis}

Thematic analysis (Braun \& Clarke, 2006) was used to analyse the interviews conducted with the therapists and the family. Initial codes were identified by Researcher 1 and an external analyst and discussed with the research supervisor, Researcher 2. Following this, a number of themes were identified and refined in comparison to the data. Themes then underwent a process of validation whereby the 2 therapists and the family members participating in this research were given an opportunity to provide feedback. The family members chose not to give feedback, and the implications of this are outlined in the 'strengths and limitations' section of the discussion.

\section{Therapy Process}

The treatment process involved engaging with culture, which is described in more detail in a paper by Malik and Mandin, (2012) as well as using general systemic ideas and techniques to explore the family and cultural systems. Malik and Mandin have argued that in order to engage with and reach an understanding of the interpersonal and family stories, one has to first engage with the often highly polarised systems of the wider professional and child protection cultural context as well as the family cultural context, and to engage with deeply held cultural beliefs, be they 'honour' or anything else. Only then with such a macro - sociocultural and micro - familial and individual alliance is a meaningful intervention that incorporates general cultural differences as well as the particulars of that case possible. The work with this family involved different permutations and combinations. Sessions took place around every two weeks initially, with greater intervals of up to six weeks nearer to the end of treatment. The main interventions were to build an alliance and develop trust with this family who felt discriminated against, and to challenge their minimising of the challenges they had faced and their cultural beliefs around gender and sexuality. All of this work was done with the aim of helping them integrate the different cultural contexts and systems that they lived in and to help them reunite as a family following the deep splits and fragmentation that resulted from the challenges they had faced. The work enabled the parents to acknowledge the impact of their behaviour on the family and vice-versa, and helped the 
youngest daughter to integrate her identity so that she could be both challenging of some of the families cultural beliefs and double standards when it came to boys and girls, whilst also loving and respecting them.

\section{Results}

Firstly, the two significant moments used as part of the IPR method are described in brief. Secondly, the results of the thematic analysis are presented in relation to four themes : (1) Inviting and accepting difference, (2) Respectfully challenging and encouraging reflection, (3) Conveying sensitivity and respect, and (4) Feeling secure in one's own cultural positioning as a therapist. These themes reflect the factors that both the family and therapists felt to be important in relation to building and maintaining a positive therapeutic alliance with BME families when taking steps to respectfully challenge core cultural beliefs.

\section{Significant moments}

The first significant moment depicted an interaction that took place around 4 months into therapy involving Therapist 1, the father and daughter and involved a conversation about prostitution and the concept of freedom. During this conversation Therapist 1 took steps to challenge the father's negative and stereotypical beliefs about the dangers of allowing young girls to have freedom within the UK by encouraging him to consider less extreme viewpoints to reach a more balanced perspective. When the father reacted defensively to her initial comments, the therapist invited him to reflect upon the ways in which his cultural values might leave him inclined to idealise Pakistan, and proposed the idea that there could also be negative social influences affecting young girls in Pakistan. The episode concluded with the father stated that he sees the therapist as "like a sister" and expressed that he feels able to talk openly and honestly with her.

The second significant moment took place later in therapy and involved the mother, father, son, daughter and both therapists. During the selected segment the therapists took steps to encourage the parents to reflect on the approach they took when raising their children, by talking about the parents' long working hours and efforts to better the family financially, and how this might have impacted on the amount of time and attention given to the children. The therapists also encouraged the parents to think about how these parenting strategies might relate to cultural values and norms in the way of maintaining a degree of pride and family status.

\section{Theme 1. Inviting and accepting difference.}

I really believe you have to start with where people are at, but it takes time to make shifts and changes, it doesn't just happen overnight. So, it's not to say it's okay, but it's just being aware of the complexity of what you're dealing with, and how these are generations of patterns, and that to change them really requires a lot of hard work on your part and on the family's part. (Therapist 1) 
This excerpt demonstrates Therapist 1's emphasis on the importance of beginning with a family's starting point before working to make gradual changes over time, rather than expecting families to be able to immediately consider an alternative perspective. Accepting and tolerating that the family have a particular viewpoint, however distinct it may be from the therapist's own, is critical when building an alliance in order to enable the family to feel heard and understood, and to better enable the therapist to begin to unpick the story and explore areas where there is room for change.

We get along so well, my dad can speak to her in our language, which is easier for him to express his emotions, because that's his first language. It's just, you know, it's really helpful. (Farah, Daughter)

Therapist 1's willingness to communicate with the family in their first language of Urdu made a difference, as highlighted by the daughter in this excerpt. Therapist 1 also emphasised how Therapist 2's acceptance and respect for the family's preferred language, and the patience that he demonstrated during these early interactions, appeared to help the family to feel at ease with their preferred method of communication, and encouraged them to open up about their experiences in a way that felt most comfortable for them.

Theme 2. Respectfully challenging and encouraging reflection.

An example discussed during the interview related to the second significant moment, in which the father had expressed a dominant view in accordance with his cultural and religious beliefs that their difficulties had been due to 'God's will', and had implied that there was nothing he could have done to change it. During the interview, Therapist 1 explained how she initially validated his belief and expressed some agreement that destiny and God's will may have played a part in his circumstances and impacted upon on subsequent events. Approaching the conversation in this way left her able to then tentatively propose that there may have been other contributing factors and lessons to be learnt from the events that unfolded - i.e. the losses of migration and the challenges of parenting and helping children to bridge different cultural contexts. When reflecting on this moment, Therapist 1 described how she had explored her own relationship to cultural and religious beliefs, and offered the father an alternative view point to his fatalistic thinking for him to consider, which challenged his perspective and encouraged him to examine this new idea without dismissing his perspective altogether. It could be judged that by tolerating and accepting the father's initial thoughts, Therapist 1 provided the father with a space to air his views within the context of a safe and welcoming alliance, and left room for these views to later be challenged and explored in a curious rather than judgemental way from within his cultural and religious system of meaning.

When we come here, I just feel like we're walking into our own living room. If feels like they kind of, just, get it? It's easy. (Farah, Daughter)

From the daughter's perspective, it appears that she and her family felt able to express their views with a feeling of comfort and security, with little fear of judgement. The daughter 
likened the therapy room to her home environment and expressed the ease in which her views could be communicated and understood by the therapists. This, together with the father's willingness to disclose information about his views and attitudes, implies that the family felt secure enough in the alliance to discuss topics of a personal nature at ease, and suggests confidence in the therapists' ability to handle this information sensitively.

We always try to bring people back to the middle ground, to the complexities. ... The father is often stuck and it's really hard to know how to manage it because if you try to point it out or to challenge it, like Therapist 1 was saying you [worry about] appearing rude. But if you don't challenge it then things don't change. And it's a really fine line. (Therapist 2)

Therapist 2 described the importance of taking steps to gently point out and challenge unhelpful or extreme views in order to help families reach a more balanced perspective and encourage long term change. With this family, the work focused on helping the father to negotiate his thoughts in relation to the different cultural contexts he was operating in, and to facilitate small adjustments in his attitudes and behaviour over time in order for positive change to occur. Therapist 2 described the intricacies of the work, and his attempts to remain careful and tactful when selecting moments to intervene to avoid the family feeling under scrutiny.

The family members spoke in their interviews about what it felt like when the therapists tried to encourage reflection.

It kind of opened up our minds. We know British culture, and we know Pakistani culture, and we know where the balance is, where the balance isn't. I think it helps him to rethink about those situations, how he could maybe be a bit less strict, or be a bit more strict. (Farah, Daughter)

Because, what she's trying to tell us, that you are in this society, where the values are different, back home is different, so if you balance then you don't face consequences or difficulties. (Sajid, Father)

The daughter's comments imply that she found it helpful to be guided towards a more integrated understanding of how to find a British-Pakistani cultural balance, particularly to enable her father to think more about the impact of cultural values on parenting. In terms of engagement, the family's ability to take on board what had been said, particularly the more critical aspects of the therapists' comments, implies that within a secure enough therapeutic alliance, core beliefs and values can be respectfully challenged, and interpreted by families as beneficial.

No matter how critical the therapists get, we will always try and take it on board, try and improve on ourselves. Cos, at the end of the day, we're here for them to be critical. If they were too nice to us, it wouldn't work! But we need them to criticise what we do, and help us make it better. (Farah, Daughter) 
Theme 3. Conveying sensitivity and respect.

For the daughter, the manner in which ideas were put across was highly important; evident in this extract where she discussed the effects of the therapists' approach.

And the way she does it, the way she portrays herfeelings and her thoughts and her emotions makes us realise so much, like sometimes, the rhetorical questions that they ask us makes us think so much, like it's a joke how much I go home and think, like, what I, what we could be doing better. (Farah, Daughter)

Later in the interview, when asked directly about the qualities of the therapists that helped the family to feel that they could engage well with their ideas, the daughter spoke at length about the therapists' approach with an air of awe and admiration.

Part of the reason is that both of them are so gentle, so kind, like even in that video clip you can see the volume of his voice, and how it relates to our volume, and how appreciative we are of his sensitivity towards us. (Farah, Daughter)

According to the daughter, the sensitive and thoughtful manner of both therapists when approaching difficult topics enabled her and her family to build a positive therapeutic alliance with both therapists and engage well in therapy. She picked up on the finer aspects of the interactions, identifying factors like tone of voice and the way the therapists provided clear and methodical explanations of the thought processes that led them to make certain comments as key. This implies that to develop a strong alliance, the manner in which core beliefs and ideas are challenged is of utmost importance when demonstrating respect for the family's position and their defining values, and that this is as much an embodied communication as verbal.

Theme 4. Feeling secure in one's own cultural positioning as a therapist.

I used [my cultural position] when engaging with families, and in the beginning it used to be harder because I'd feel trapped in that position? Whereas now I feel like I'm able to use that to engage but also feel more confident with my professional identity to challenge families, as well. I kind of do that insider-outsider dance? (Therapist 1)

To respectfully challenge core beliefs required confidence on the therapists' part, and a security in their own sense of identity in relation to the areas that their own cultural beliefs matched and differed from the family. The positive alliance that developed with the family appeared to be aided by the fact that Therapist 1 shared similar religious beliefs, and had inside knowledge and experience of the Pakistani culture through her own family of origin. Her cultural position not only increased her awareness of the common views and stereotypes that may be held by somebody of the father's background, but she was also able to respectfully challenge his perspective through her knowledge of how her own personhood, 
subjectivity and beliefs are culturally constructed, and in light of her additional experience of navigating the British culture.

Partly because she is from the background that we're from. It's dead easy. It's like looking back to her own family, if they had problems, what did she do wrong, what could she do better. (Farah, Daughter)

Therapist 2 explained that building a therapeutic alliance with a family of a different cultural background can be difficult during the initial stages, and that working alongside a therapist of the same cultural background as the family facilitated this early engagement process. The therapists felt that by observing a positive working relationship between somebody of a British-Pakistani Muslim background (Therapist 1) and somebody representing the whitedominant culture (Therapist 2) the family were able to learn to trust Therapist 2 and open up to him more and more over time.

\section{It helps because Therapist 1 brings that knowledge from within, and I bring that naivety, ignorance and knowledge from the White dominant [culture] (Therapist 2)}

Therapist 2 spoke about the importance of connecting with the family on an emotional level, and how by taking the time to remain attuned to aspects of his own story that resonated with the family's, such as experiences of loss or injustice, he was able to connect more closely with some of the family's experiences. Therapist 1 emphasised the way that Therapist 2 overtly respected her ease of communication with the family, particularly at moments when the family preferred to speak in Urdu. She also highlighted how Therapist 2's patience and open-minded nature facilitated the building of the therapeutic alliance.

I don't think I could just do this work with just anybody, I felt like he had the right qualities because he wouldn't jump to conclusions with families, that he would have the patience to remain curious, and not make a condemning judgement. (Therapist 1)

\section{Discussion}

This research focused on investigating the ways in which family therapists built and maintained a positive therapeutic alliance with a family of a black and minority-ethnic (BME) background whilst taking steps to challenge the family's core cultural and religious beliefs. Accepting and tolerating points of view which may or may not have matched the therapists' own was thought to help the family feel at ease and better able to express their views in a non-judgemental and containing environment. The way the therapists encouraged reflection and consideration of differing viewpoints was received positively by the family, particularly due to the sensitive and respectful manner in which core beliefs were challenged and alternative ideas proposed. Finally, the therapists felt that their awareness of their own cultural construction and positioning and the differing degrees of insight they each had into the family's culture helped the family to respond well to the curious and exploratory approach that was taken when discussing core belief systems. 
As previously outlined, challenging clients' beliefs in relation to their core values is often advised against in cultural work (e.g. Asnaani \& Hofmann) and has tended to be the modus operandi ${ }^{2}$, largely due to a concern that clients may perceive therapists as disrespectful or rude (Hays, 2009). Interestingly, the findings of this research appear to suggest that such challenges are possible, if handled in an appropriate way. Not only were core beliefs effectively challenged by the therapists, but the family also reported a satisfaction in the way that criticisms were delivered and received. The daughter spoke of her appreciation of the therapists' efforts to help the family re-evaluate their attitudes and values, outlining the benefits of this for both herself and her parents when thinking about how to integrate their cultural values. Thus, if the alliance is good enough, this position can be experienced as helpful by the client. Drawing on dialogical therapy ideas, Bertrando (2007) similarly argues that it can be helpful for the therapist to express an opinion, as it is only then that a true dialogue in which his ideas are put into play with that of the client can take place. This in effect could be seen as a less colonising starting point as the both the client and therapists take a subjective position (Krause, 2012). We do not wish though to oversimplify or suggest that changing deeply held cultural beliefs is easy, and no doubt the pressure of the child protection context placed greater pressure on this particular family, but with a good alliance and engagement then this is possible.

\section{A sensitive approach to difference}

A number of factors were thought to aid the development of a strong therapeutic alliance and enable the therapists to feel able to challenge the family's core beliefs. Demonstrating support for the family's culture-related stressors and their resulting beliefs by accepting their perspective appeared to facilitate the discussion of sensitive issues, something that is considered to be fundamental when building a positive alliance with BME clients (Vasquez, 2007). Krause (2012) argues that constructive and effective communication will only take place if we are able to acknowledge others' perspectives and develop an understanding of the history, beliefs, motivations, wishes and intentions of clients and ourselves, and Friedlander (2006) highlights the need to remain aware of values important to the family in relation to their cultural context. Both therapists took time to explore the values important to the family, and respectfully acknowledged significant issues such as pride, achievement and status. Adopting an empathic and gentle approach to conversations was also identified by both the family and the therapists as a key element of engagement, and is thought of as an important component of a positive therapeutic alliance with any client group (e.g. Asnaani \& Hofmann, 2012). The therapists' approach appeared to help foster the growth of mutual trust and respect between themselves and the family members, factors important for building an alliance and strengthening the quality of the therapeutic relationship (Horvarth et al., 2011; Horvath \& Bedi, 2002). Demonstrating care for family members and providing a safe space for individuals to share their views have been highlighted as particularly important when engaging BME families (Pandya \& Herlihy, 2009), and in this case appeared to contribute

\footnotetext{
${ }^{2}$ As was also highlighted in the Monroe enquiry into the Victoria Climbie case.
} 
towards the development of a secure enough relationship through which core belief systems could be challenged.

\section{Awareness of one's own cultural identity}

Having a keen awareness of one's own positioning and how this may impact on relationships with clients is another factor to consider when working across- and within-cultures (Plummer, 1997). Some evidence suggests that clients' perceptions of a therapist's high degree of cultural competence can also enhance therapeutic alliance (e.g., Owen et al., 2010), something that the daughter highlighted as a factor contributing towards her warm relationship with the female therapist. The therapists' insight into their own cultural construction and racial identity, and the application of this knowledge when finding ways of identifying with the family and showing an understanding of their circumstances, as well as challenging them, was seen as helpful, particularly as the therapists were aware of the ways in which their background differed and matched the family's and were able to adjust their approach accordingly. This family, like many BME families, had experienced racism (and other forms of discrimination including sexism and classism) from social institutions, which set the context for the therapeutic encounter. Rober (2010) acknowledges this by arguing that our own voices, empowered by the dominant culture, have the potential to colonise.

However, we would like to stress that ethnic matching alone is not sufficient to build a robust therapeutic alliance, but that it requires awareness of one's own culture and the ability to take a critical stance, and to use these points of similarity and difference to engage with the family whether the therapist is white or from a BME background.

Therapist 1 spoke of her approach when initiating challenging conversations with the family, and the direct nature of her criticisms when discussing culturally-related views. Sharing a similar background to the family as a British-Pakistani Muslim may have led her comments to be received as less threatening, and her knowledge of the family's culture may have given her more confidence when evaluating appropriate moments to intervene. Despite these cultural similarities though, it is likely that the manner in which Therapist 1 approached these subjects and the tactful nature of her interventions had an influence on the family's willingness to accept her comments. Knowledge of typical cultural and familial patterns and the problems that may occur as a result of events like migration is thought to be helpful but not sufficient when building alliances with BME clients (Malik \& Mandin, 2012; Sue \& Zane, 2009), as knowledge must be applied strategically and conveyed in a sensitive manner with a degree of authenticity and curiosity.

Therapist 2 also took steps to challenge the family, actions that the family referred to as helpful and thought-provoking. His style was perceived as gentle and warm, and Therapist 1 emphasised the positive effects of his ability to remain patient and avoid jumping to negative conclusions. It could be that Therapist 2 adopted a 'one-down' position (Fisch et al., 1975) as a demonstration of respect towards the family, a position that also implies an understanding of Therapist 1's position as the clinician holding most of the power and cultural knowledge in this particular case. As Therapist 2 suggested, involving a therapist from a similar 
background is likely to have eased the initial engagement and helped this family to settle into therapy, whereas the other factors discussed appeared to have played a central role in strengthening and maintaining the alliance.

It is also important to consider that the alliance between the two therapists and their ability to work jointly in an effective manner was also fostered in the context of a mainstream CAMHS that had consciously decided and committed to engage with BME communities by initiating the MCTC, a specialist cultural service (Malik, 2009). The integration of the cultural service over eighteen years occurred through joint working of clinicians on complex cases and relationship building through organisational reflexivity. As part of the work, the MCTC had also engaged in building alliances with community organisations and through this had built knowledge and relationships which made for a strong foundation for engaging with BME clients.

\section{Strengths and limitations}

This is the first known study to investigate closely the process of building and maintaining an alliance with BME families from both a therapist and client perspective. Combining qualitative interviewing methods with IPR has provided a multifaceted account of the experience of challenging core beliefs, and examining the case of only one family provided an opportunity to delve deeper into the analysis and capture the knowledge and experience of both therapists that was demonstrated through this select piece of work. The diverse cultural backgrounds of the therapists, researchers and family has brought a richness to the research, and allowed the work to be interpreted from multiple viewpoints.

To facilitate an in-depth analysis, two interviews were conducted to explore the case of one family, meaning that the rule of data saturation was not used. Care must therefore be taken when considering the findings in relation to other families. Only two family members were available to be interviewed during the period requested, as the other family members were temporarily abroad. It should also be noted that the findings of this research focus largely on the therapists' strengths when engaging BME families in therapy, as opposed to addressing areas for change or improvement. The family portrayed the therapists in a highly positive light and were reluctant to criticise their approach, perhaps due to a strong loyalty towards the therapists and an appreciation of the work that had taken place. This might also tie in with the significance commonly given to the value of respect for authority and status within Asian communities, as criticising the therapists (who had been referred to as "father" and "mother" figures) may have been seen as a form of disrespect. Throughout the process of interviewing, the researcher was mindful of her own position as a white British female representing a majority-group professional, and considered whether this may have led the family to restrict or censor some information. The family may not have felt comfortable to disclose some of the more critical aspects of their treatment, and could have been keen to present a positive impression by maintaining their satisfaction with the service. 


\section{Implications for Practice}

This research implies that if cultural topics are approached in sensitive and respectful manner, by trusted therapists with a degree of knowledge and awareness of a family's culture as well as their own cultural position, then the challenging of core beliefs can be an effective way to facilitate gradual change and further strengthen the therapeutic alliance with BME families. We argue that in order to build a genuine alliance and engage with culture it is vital for the therapist to feel able to respectfully challenge core beliefs, but this is more effective if it is done from within the client's meaning-making system. It is also important to be aware that each individual's cultural context will vary, and that maintaining a degree of flexibility is important when judging the appropriate approach and timing of a particular intervention (Kirmayer, 2012).

This process requires a degree of cultural reflexivity, and also applies to organisations when developing authentic alliances with BME practitioners and communities. Shying away from such conversations in practice, or defensively withdrawing from difficult discussions relating to cultural belief systems could instead create distance between client and therapist, and could hinder both the process of change and the development of a positive therapeutic alliance. We believe that this modus operandi has contributed to the contemporary context in which mainstream institutions have failed to engage BME communities or issues on a number of high profile cases (e.g. the Rotherham child exploitation sex scandal and the Trojan horse affair in schools), and that this has led to the punitive backlash by politicians and the media that professionals have been 'too culturally politically correct' ("Rotherham Scandal", Guardian Newspaper, Sep 2014). Rather, we think that there has not been a sufficient genuine two-way engagement process with BME clients and communities to build alliances and facilitate change.

The ideas that have arisen from this research may be relevant not only to therapeutic work with families, but similarly to individual and group interventions with minority populations. The findings may also be applicable to professionals involved in work with BME clients within the mental health or healthcare sectors such as social services, as a large proportion of the work requires the ability to successfully engage clients and maintain a positive therapeutic alliance despite conflict or differing views, whilst facilitating effective change. 


\section{References}

Asnaani, A., \& Hofmann, S. G. (2012). Collaboration in Multicultural Therapy: Establishing a Strong Therapeutic Alliance Across Cultural Lines. Journal of Clinical Psychology, 68, 187-197.

Beck, J. S. (2005). Cognitive Therapy for Challenging Problems. New York: Guildford Press.

Bertrando, P. (2007). The Dialogical Therapist. London: Karnac

Braun, V., \& Clarke, V. (2006). Using Thematic Analysis in Psychology. Qualitative Research in Psychology, 3, 77-101.

Elliott, R., Bohart, A. C., Watson, J. C., \& Greenberg, L. S. (2011). Empathy. In J. C. Norcross (Ed.), Psychotherapy Relationships that Work (2nd ed.). New York: Oxford University Press.

Fisch, R., Weakland, J. H., Watzlawick, P., Segal, L., Hoebel, F. C., \& Deardorff, C. M. (1975). Learning brief therapy: An introduction manual. Palo Alto, CA: Mental Research Institute.

Friedlander, M. L., Escudero, V., \& Heatherington, L. (2006). Therapeutic Alliances in Couple and Family Therapy: An Empirically Informed Guide to Practice. American Psychological Association.

Hays, P. A. (2009). Integrating Evidence-Based Practice, Cognitive-Behavior Therapy, and Multicultural Therapy: Ten Steps for Culturally Competent Practice. Professional Psychology: Research and Practice, 40, 354-360.

Hofmann, S. G. (2006). The Importance of Culture in Cognitive and Behavioral Practice. Cognitive and Behavioral Practice, 13, 243-245.

Horvath, A. O., \& Bedi, R. P. (2002) The alliance. In J. C. Norcross (ed.) Psychotherapy Relationships That Work: Therapists' Contributions and Responsiveness to Patients (pp. 37-69). New York: Oxford University Press.

Horvath, A. O., Del Re, A. C., Flückiger, C., \& Symonds, D. (2011). Alliance in individual psychotherapy, 48 (1), 9-16.

Kagan, N. (1980). Influencing Human Interaction - Eighteen Years with IPR. In A.K. Hess (Eds.), Psychotherapy Supervision: Theory, Research and Practice (pp. 262-283). New York: Wiley.

Kirmayer, L. (2007). Psychotherapy and the cultural concept of the person. Transcultural Psychiatry, 44, 232-257. 
Kirmayer, L. (2012). Cultural competence and evidence-based practice in mental health: Epistemic communities and the politics of pluralism. Social Science \& Medicine, 75, 249-256.

Krause, I. B. (2012). Culture and Reflexivity in Systemic Psychotherapy: Mutual Perspectives. London: Karnac.

Lee, E. (2011). Clinical Significance of Cross-Cultural Competencies (CCC) in Social Work Practice. Journal of Social Work Practice, 25, 185-203.

Malik, R., ,Fateh, R., \& Haque, R. (2009). In Fernando, S. \& Keating, F. Mental health in a Multi-ethnic Society: a Multidisciplinary Handbook ( $2^{\text {nd }}$ ed.). Routledge

Malik, R., \& Mandin, P. (2012). Engaging within and across culture. In Krause, I. B. (Ed.), Culture and Reflexivity in Systemic Psychotherapy: Mutual Perspectives (pp.201222). London: Karnac.

May blames 'institutionalised political correctness' for Rotherham scandal". (The Guardian, 2014). Retrieved from http://www.theguardian.com/uk-news/2014/sep/02/theresamay-political-correctness-rotherham-abuse.

Muslims must embrace our British values, David Cameron says. (The Telegraph, 2011). Retrieved from http://www.telegraph.co.uk/news/politics/davidcameron/8305346/Muslims-must-embrace-our-British-values-David-Cameronsays.html.

Owen, J., Leach, M. M., Wampold, B., \& Rodolfa, E. (2011). Multicultural approaches in psychotherapy: A rejoinder. Journal of Counseling Psychology, 58(1), 22-26.

Pandya, K., \& Herlihy, J. (2009). An exploratory study into how a sample of a British South Asian population perceive the therapeutic alliances in family therapy. Journal of Family Therapy, 31, 384-404.

Plummer, D. L. (1997). A Gestalt Approach to Culturally Responsive Mental Health Treatment. Gestalt Review, 1, 190-2014.

Rober, P., \& Seltzer, M. (2010). Avoiding Colonizer Positions in the Therapy Room. Family Process, 49, 123-137.

Sue, S., \& Zane, N. (2009). The Role of Culture and Cultural Techniques in Psychotherapy: A Critique and Reformulation. Asian American Journal of Psychology, 1, 3-14.

Tseng, W. (1999). Culture and Psychotherapy: Review and Practical Guidelines. Transculture Psychiatry, 36, 131-179.

Vasquez, M. J. T. (2007). Cultural Competence and Evidence-Based Practice in Mental Health Services: A Complementary Perspective. American Psychologist, 62, 563-574. 
Wood, P. S., \& Mallinckrodt, B. (1990). Culturally Sensitive Assertiveness Training for Ethnic Minority Clients. Professional Psychology: Research and Practice, 21, 5-11. 